\title{
A single centre, double arm, triple-blind randomised controlled trial comparing the efficacy of combined letrozole and clomiphene versus the only letrozole as a method of ovulation induction in women with polycystic ovarian syndrome.
}

\author{
Soumya Panda ${ }^{1}$, Sharmila $V^{1}$, Vinoth Kalidoss ${ }^{1}$, and Smrutismita Hota ${ }^{1}$ \\ ${ }^{1}$ AIIMS Mangalagiri
}

June 25, 2021

\begin{abstract}
Objective: To evaluate the efficacy of combined therapy of letrozole and CC compared to the use of letrozole alone to achieve ovulation in infertile women with PCOS. Design: This was a single-centre, double arm, triple-blind randomized controlled trial. The study was conducted after taking approval from Institutional Ethics Committee and was prospectively registered with the clinical trials registry- India. Setting: Women were randomly assigned to receive a combination of $2.5 \mathrm{mg}$ letrozole and placebo daily or a combination of $2.5 \mathrm{mg}$ letrozole and $50 \mathrm{mg}$ CC daily on cycle days 3-7 for one treatment cycle. Methods: 80 participants were enrolled and randomized to either the letrozole-only group $(n=40)$ or the combined letrozole and CC $\operatorname{group}(n=40)$. Main outcome measures: The primary outcome measure was documentation of ovulation, confirmed by TVS 48 hours later with or without serum progesterone estimation.Results: In comparison, both Intention to treat (ITT) and perprotocol (PP) analysis showed a statistically significant increase in ovulation rate in the arm treated with combined letrozole and CC. Conclusion: Combination therapy with letrozole and clomipheneshould be considered as a first-line ovulation induction agent for infertile patients with PCOS owing to its low cost and better tolerability. Additional well designed clinical trials are required to determine the efficacy of the combination of letrozole and $\mathrm{CC}$ in terms of clinical pregnancy rates and live birth rates. Funding: There is no funding agency for this study. Keywords: Clomiphene, Letrozole, Polycystic ovarian syndrome, ovulation induction
\end{abstract}

\section{Introduction:}

With an estimated prevalence of $5 \%-10 \%$ in the general population, polycystic ovary syndrome (PCOS) is by far the most common cause of anovulatory infertility.[1] Clomiphene citrate (CC) is a commonly prescribed pharmacologic agent used to induce ovulation in women with PCOS because of its antiestrogenic property. It acts by inhibiting the negative feedback of estrogen resulting in increased secretion of gonadotropin hormones which induces ovarian follicular growth.[2,3] Over the last decade, letrozole has become a popular oral ovulation induction agent. It works by inhibiting the aromatase enzyme and thereby preventing the conversion of androgen to estrogen. The suppressed estrogen production inhibits negative feedback on the hypothalamus and increases the secretion of FSH. The increased follicular sensitivity to FSH leading to a transient increase in intraovarian androgens is thought to be associated with improved ovulatory rates with the use of letrozole.[4] Apart from letrozole or CC, options for ovulation induction in PCOS patients are limited and thus leaving the use of gonadotropin injections as the only option of ovulation induction in these patients. Unfortunately, this option of treatment is available only at a higher cost and also with a risk of ovarian hyperstimulation. Due to the different mechanisms of action, it has been postulated that combining letrozole and CC may result in an improved ovulatory rate and thus could be a potential option of ovulation induction in PCOS women before proceeding to the use of gonadotropins. To date, there are only rare 
clinical trials that have studied the effects of combined letrozole and CC on ovulation rate or pregnancy rate.[5] Therefore, we conducted this randomized controlled trial with clearly stated study endpoints to test the hypothesis that combined therapy of letrozole and CC is effective and superior to the use of letrozole alone to achieve ovulation in women with PCOS.

\section{Objective:}

The primary objective was to evaluate the efficacy of combined therapy of letrozole and CC compared to the use of letrozole alone in terms of ovulation rate in infertile women with PCOS. Secondary objectives were to characterise the side-effects profile and evaluating the ultrasonographic cycle characteristics on this treatment regimen.

\section{Methodology:}

\section{Study Design:}

This was a single-centre, double arm, triple-blind randomized controlled trial. The study was conducted at All India Institute of Medical Sciences, Mangalagiri, India after taking approval from the Institutional Ethics Committee (IEC/AIIMS/MG/2020/32). The study was prospectively registered with the clinical trials registry- India CTRI/2020/09/028012. The study is reported following the Consolidated Standards of Reporting Trials (CONSORT) guidelines.[6]

\section{Participants 83 Setting :}

The study was conducted from October 2020 to May 2021 at a tertiary care teaching institute in India. Eligible participants were women aged 20-35 years with anovulatory infertility due to PCOS diagnosed by Rotterdam's criteria which entails any two of the following finding (i) ovulatory dysfunction (ii) clinical or biochemical features of androgen excess and (iii) polycystic ovarian morphology on ultrasound.[7] The threshold for defining polycystic ovarian morphology was [?] 12 follicles of $10 \mathrm{ml}$. The participants, the consultant who performed transvaginal ultrasound (TVS) for outcome assessment and the data analyst were blinded to treatment allocation.

\section{Intervention:}

Randomization was done during the first 3 days of spontaneous menses or while taking medroxyprogesterone (10 mg twice for 5 days) to induce withdrawal bleed. Women were randomly assigned to receive a combination of $2.5 \mathrm{mg}$ letrozole and placebo daily or a combination of $2.5 \mathrm{mg}$ letrozole and $50 \mathrm{mg}$ CC daily on cycle days 3-7 for one treatment cycle.A baseline scan was done on day- 2 or 3 of the menstrual cycle. The growth of follicles was monitored by performing transvaginal sonography (TVS) on day 11 of the cycle and the scan was repeated afterwards depending on the follicular growth. All the scans were performed by a single consultant using a transvaginal probe (frequency of 3-10 MHz) of the diagnostic ultrasound system (Ecube i7, Alpinion medical systems). Injection human chorionic gonadotropin 5,000 unit was given after at least one follicle reached a size of $18 \mathrm{~mm}$ diameter. Timed intercourse was advised 24 hours to 36 hours after hCG injection. Ovulation was confirmed by TVS 48 hours later with or without serum progesterone estimation. Those who conceived after treatment were followed up at our hospital and their pregnancy outcomes were tracked with regular follow up visits. All the clinical decisions were taken with the mutual agreement of two consultants.

\section{Inclusion criteria:}

Infertile Patients attending our OPD were included in our study if the following inclusion criteria were fulfilled.

1. The age of the patient is between $20 \mathrm{yrs}$ to $40 \mathrm{yrs}$

2. Diagnosed as PCOS according to Rotterdam criteria.

3. a normal hysterosalpingogram or laparoscopy has been documented;

4. no recorded history of pelvic surgery and/or pelvic inflammatory disease; 
5. Male partners having a normal semen analysis, according to the 2010 World Health Organization criteria, within the preceding 6 months and

6. no history of treatment with exogenous gonadotropin and not on treatment with metformin.

Exclusion criteria: The exclusion criteria were-

1. Endocrine factors other than anovulatory PCOS like hyperprolactinemia, hypothalamic amenorrhea, premature ovarian failure and ovarian tumour or clinical suspicion of other etiologies that mimic PCOS warranting additional evaluation.

2. Uncorrected thyroid disease; uncontrolled type 1 or 2 diabetes mellitus or hypertension.

3. Allergy or contraindications to letrozole or CC.

4. Other associated factors of infertility like a malefactor, uterine factor and endometriosis.

\section{Outcome Measures}

The primary outcome measure was documentation of ovulation, confirmed by TVS 48 hours later with or without serum progesterone estimation.

The secondary outcomes were :

1) Size and number of developing follicles and endometrial thickness noted in TVS on the day of HCG injection;

2) Conception with the treatment cycle: a positive serum or urinary test of hCG;

3) Clinical pregnancy: an intrauterine pregnancy with fetal heart motion determined by ultrasonography;

4) multiple pregnancies;

5) pregnancy loss, including biochemical miscarriage or ectopic pregnancy; and

6) adverse events related to the medications: Adverse events are defined as any side effects that the subject recorded on her daily calendar log during the study cycle. Serious adverse events (SAEs) are defined as events that were fatal or immediately life-threatening or required inpatient hospitalization.

\section{Sample Size calculation:}

Thirty-three subjects per group were required to obtain $80 \%$ statistical power to demonstrate a clinically meaningful $34 \%$ absolute difference in ovulation rate between treatment groups, assuming a $43 \%$ ovulation rate for letrozole a two-sided significance level of 0.05 using open Epi software version 3.01. The sample size was increased to 40 per arm to allow for a dropout rate of $20 \%$. We selected a benchmark of $34 \%$ difference between groups based on a previous study that compared combined letrozole and clomiphene with letrozole alone for ovulation induction. [5]

\section{Randomization}

Randomization was done in a ratio of 1:1. We used the block randomization technique with a block size of 8 for patient recruitment. The random allocation was done using computer-generated random numbers for each block and stored in a sealed non-transparent cover. The study drug and placebo were coded as A and B. A nursing officer was assigned to distribute the drugs based on the participant's allocation. The study drug and placebo were matched for color, shape, and size. The allocation scheme was not disclosed to the patients, investigators, and data analyst.

Statistical analysis:

All the analysis was performed by using Statistical package for social sciences (SPSS) software (version 21.0). Analysis was based upon intention to treat (ITT) as well as per-protocol (PP). ITT analysis included all participants who were randomized, irrespective of whether or not they received the study drug. Per protocol, analysis included only those participants who completed the study after randomization. Participants who were lost to follow-up were assumed neither to have ovulated nor to have conceived in the ITT analysis. For 
categorical variables, either chi-square or Fisher exact test was used at a two-sided significance level of 0.05 for testing differences between the two treatment groups. For continuous variables, the mean \pm SD in each group was reported. Student t-test was used to analyze normally distributed data and in the case of skewed data, the median and interquartile range are reported and Mann-Whitney U tests were applied. A subgroup analysis was performed to assess ultrasound characteristics among those who ovulated.

\section{Results:}

\section{Characteristics of the Participants:}

A total of 118 participants were assessed for enrolment. Of these, 38 were excluded (34 not met inclusion criteria and 4 declined to participate). The remaining 80 participants were enrolled and randomized to receive either the letrozole $2.5 \mathrm{mg}$ along with a placebo $(\mathrm{n}=40)$ or a letrozole $2.5 \mathrm{mg}$ and CC $50 \mathrm{mg}(\mathrm{n}=40)$. After randomization, three participants were lost to follow up because of personal reasons leaving 77 participants that took the allocated treatment. Figure 1 presents a Consolidated Standards of Reporting Trials (CONSORT) flow diagram for this study.[6] The first enrolment was in October 2020 and the last enrolled participant finished the study medication in May 2021. Follow-up for the study cycle outcomes was completed in June 2021. As summarized in Table 1, there were no significant differences between the two groups as far as the baseline characteristics are concerned.

\section{Primary Outcome}

As depicted in Table $2 \& 3$ women who received both letrozole and CC had a higher ovulation rate than women receiving letrozole and placebo in both the ITT and PP analyses. On comparison of letrozole and CC group versus letrozole and placebo group in the ITT analysis, we found an ovulation rate of $72.5 \%$ versus $37.5 \%$, P-value 0.0032 , the rate ratio for ovulation with combination treatment $1.93,95 \%$ CI 1.24 to 3.01 (Table 2). Similarly, PP analysis showed an ovulation rate of $74.35 \%$ versus $39.4 \%$, P value:0.0027, the rate ratio for ovulation with combination treatment $1.88,95 \%$ CI 1.2196 to 2.9095 for the respective groups Table 3).

\section{Secondary Outcomes}

There were no statistically significant differences in conception or clinical pregnancy rates. Much larger sample size would be needed to detect differences in these outcomes (Table $2 \& 3$ ). There were no multiplegestation pregnancies in either group. Among those who conceived, the rates of pregnancy loss were similar in the two treatment groups (Table $2 \& 3$ ). Cycle characteristics are presented in Table 4. Among the participants as well as those who ovulated there was no significant difference in endometrial thickness or the number of follicles $>15 \mathrm{~mm} /$ Cycle between the two groups.

\section{Adverse Events}

No SAEs related to the treatments occurred during the study. Participants in both groups reported similar acceptability of side-effects. None of the patients was hospitalised or seek any additional care during the treatment month for any of the experienced adverse effects (Table 5). The difference in the side effects profile between the two groups was statistically not significant. The most commonly reported side effects in the letrozole and placebo group included headache (25.6\%), abdominal bloating (17.9\%) and fatigue (15.3\%). On the other hand, the most commonly encountered side effects in the letrozole and CC group included: headache $(28.9 \%)$, abdominal bloating $(21 \%)$ and hot flashes $(15.6 \%)$.

\section{Discussion:}

The results of this study support our hypothesis that a combination of letrozole and CC is superior to letrozole alone for achieving ovulation in infertile women with PCOS. This study found that the combination of letrozole and CC had a higher ovulatory rate, with a 35\% absolute difference in favour of this treatment group. Conception, pregnancy, pregnancy loss, and live birth were similar in both groups. However, it should be acknowledged that the study was underpowered to detect a significant difference for these outcomes. But given the absence of prospective well-controlled clinical trial data on the combination of letrozole and CC, 
the most practical and feasible first step would be to assess the ovulation rate in a well designed randomized controlled trial.

Both Letrozole and clomiphene are commonly used, ovulation-inducing agents. [2,3,8] The long half-life of CC (around two weeks) and its antiestrogenic effects on endometrial development and cervical mucus production, have been blamed to contribute to a relatively low pregnancy rate despite possessing a high ovulation rate.[2,3] The initial concerns of congenital anomalies associated with the use of letrozole have been mitigated by two large multicentric studies.[9,10] Letrozole has been considered as a potentially better alternative for ovulation induction as it doesn't block estrogen receptors in both central and peripheral target tissues, thus normal central feedback mechanisms remain intact.[11] Moreover owing to a short half-life (48 hours) it is cleared from the circulation rapidly thus allowing the rise of estrogen later. This has a favourable effect on the endometrium and hence pregnancy rates.[4,11] Various clinical trials have compared the efficacy of letrozole and CC in infertile women with PCOS.[2,3,11-15] These data have also been analyzed in a systematic review and meta-analysis.[16,17] Franik et al.[16], found higher pregnancy and live birth rates with letrozole compared to CC. However, the quality of evidence generated was not robust enough as most of the included studies were of questionable quality owing to unclear or high risk of bias association.

Gonadotropin injections like human menopausal gonadotropins (hMG), urinary or recombinant FSH, have been used as an advanced step of ovulation induction in cases of CC or letrozole failure or resistance.[18,19] However, the high cost of gonadotropins and the risk of ovarian hyperstimulation (OHSS) especially in women with PCOS makes their use cumbersome. Hence, of late some authors advocated the combination of letrozole and clomiphene as a method of ovulation induction before proceeding to treatment with gonadotropins.

The only randomised controlled trial that compared similar treatment groups as ours was performed by Mejia et al.[5] They also found a higher ovulation rate in the combined letrozole and CC group compared to the letrozole alone group. However, in their study, they did not exclude patients who were on treatment with metformin which could have an additive effect on ovulation induction and thus has the potential to confound the result. Also, the study was not blinded to participants and the investigator. Another prospective cohort study recruited 100 patients resistant to letrozole alone (four cycles) and CC alone (six cycles) and demonstrated a follicular development rate of $82.9 \%$ (213 of 257 cycles) based on the development of a dominant follicle with the use of $5 \mathrm{mg}$ letrozole plus $100 \mathrm{mg} \mathrm{CC}$ daily for 5 days.[20] In addition, in this study patients also received an FSH injection on day 11. Although this study provided initial interesting findings regarding the use of combination treatment with letrozole and $\mathrm{CC}$, follicle size alone is not necessarily a reliable or accurate way to confirm ovulation. Again, the study used a limited population of PCOS women resistant to both CC alone and letrozole alone and was not randomized.

The cumulative ovulation over five cycles for letrozole alone (including doses of 2.5, 5.0, and $7.5 \mathrm{mg}$ ) in the PPCOS II trial was $61.7 \%$ (834 of 1,352 cycles).[11] Going by our study results, the ovulation rate in our combination-treatment group was found to be higher than the ovulation rate reported in the PPCOS II trial, suggesting that there may be a synergistic effect of the combination of these medications.[11] It will be important for future studies to compare cumulative ovulatory rate and live birth rate between letrozole alone and the combination of letrozole and CC in multiple cycles as well as with different dosing regimens.

Of note, the side-effect profiles were similar between the two arms of the present study. Furthermore, no serious adverse events (SAEs) were detected related to the study treatments in any of the treatment group. However, the study was not powered to assess a difference between groups for congenital birth defects. Similar to our study results, Meija et al., also found no difference in side-effects profiles between both groups.[5]

\section{Strengths and Limitations:}

Methodology and the study design are the primary strength of this study. We have chosen clearly stated endpoints as outcome measures. In addition, the triple blinding nature of this study adds to the strength of the study. Another strength of this study is the pragmatic approach in the eligibility criteria, providing results for a patient population that is likely similar to that in other reproductive endocrine clinics. 
Few limitations should be considered while analysing the study results. Firstly, only one treatment cycle per participant was considered and there was not a stair-step approach for increasing the dose in either treatment group. Thus, cumulative ovulation rate and effect of different combinations of doses in additional cycles of treatment could not be evaluated. However, the present study design was found to be appropriate to evaluate a relatively newer form of treatment and simultaneously provided a cost-effective and responsible method of evaluation. Secondly, we did not choose clinical pregnancy rate or live birth rate as the primary outcome measure. However, as discussed, the combination treatment with letrozole and clomiphene is a newer form of a treatment approach with very scarce available data on this approach. Hence, it was prudent to assess the ovulation rate, which would be the most practical and feasible step at this stage.

\section{Conclusion:}

The results of this novel study suggest that the combination therapy with letrozole and clomiphene offers superior ovulation rates in infertile PCOS subjects and should be considered as a first-line agent for ovulation induction in such subjects owing to its low cost, low risk and better tolerability. Additional well designed randomized clinical trials are needed to determine the efficacy of the combination of letrozole and CC in terms of clinical pregnancy rates and live birth rates. Despite having certain limitations, this randomised controlled trial carries an overall good and reproducible study design and thus has the potential to generate reliable and generalizable evidence.

Disclosure Statement : There is nothing to be disclosed for this article.

\section{Funding: None}

Author contributions: SRP contributed with conception and design, acquisition of data, analysis and interpretation of data, drafting of manuscript, critical revision of the manuscript for intellectual input, statistical analysis and supervision. SV contributed with conception and design, acquisition of data, analysis and interpretation of data, critical revision of the manuscript for intellectual input, and supervision. VK contributed with analysis and interpretation of data, critical revision of the manuscript for intellectual input, statistical analysis and supervision.SH contributed with conception and design, acquisition of data, analysis and interpretation of data, and supervision.

Ethical statement with approval date: Institutional (AIIMS, Mangalagiri) Ethics Committee approval (IEC/AIIMS/MG/2020/32) was taken on 01/09/2020.

\section{References:}

1.Hoffman BL, editor. Williams gynaecology. Third edition. New York: McGraw-Hill Education; 2016. 1270 p.

2. Roy K, Baruah J, Singla S, Sharma J, Singh N, Jain S, et al. A prospective randomized trial comparing the efficacy of Letrozole and Clomiphene citrate in induction of ovulation in polycystic ovarian syndrome. J Hum Reprod Sci. 2012;5(1):20.

3. Amer SA, Smith J, Mahran A, Fox P, Fakis A. Double-blind randomized controlled trial of letrozole versus clomiphene citrate in subfertile women with polycystic ovarian syndrome. Hum Reprod. 2017 Aug 1;32(8):1631-38.

4. PCOS_Evidence-Based-Guidelines_20181009.pdf.

5. Mejia RB, Summers KM, Kresowik JD and Van Voorhis BJ.A randomized controlled trial of combination letrozole and clomiphene citrate or letrozole alone for ovulation induction in women with polycystic ovary syndrome.Fertil and Steril 2019;111(3):571-578.e1

6. Schulz KF, Altman DG, Moher D, Group C. CONSORT 2010 statement: updated guidelines for reporting parallel group randomized trials. Open Med 2010;4:e60-8 
7. Rotterdam ESHRE/ASRM-Sponsored PCOS Consensus Workshop Group. Revised 2003 consensus on diagnostic criteria and long-term health risks related to polycystic ovary syndrome. FertilSteril. 2004 Jan;81(1):19-25. doi: 10.1016/j.fertnstert.2003.10.004. PMID: 14711538.

8. Mitwally MF, Casper RF. Use of an aromatase inhibitor for induction of ovulation in patients with an inadequate response to clomiphene citrate. FertilSteril. 2001;75:305-309.

9. Tulandi T, Martin J, Al-Fadhli R, Kabli N, Forman R, Hitkari J, et al. Congenital malformations among 911 newborns conceived after infertility treatment with letrozole or clomiphene citrate. FertilSteril. 2006 Jun;85(6):1761-65.

10. Forman R, Gill S, Moretti M, Tulandi T, Koren G, Casper R. Fetal Safety of Letrozole and Clomiphene Citrate for Ovulation Induction. J ObstetGynaecol Can. 2007 Aug 1;29(8):668-71.

11. Legro RS, Brzyski RG, Diamond MP, Coutifaris C, Schlaff WD, Casson P, et al. Letrozole versus clomiphene for infertility in the polycystic ovary syndrome. N Engl J Med 2014;371:119-29.

12. Liu C, Feng G, Huang W, Wang Q, Yang S, Tan J, et al. Comparison of clomiphene citrate and letrozole for ovulation induction in women with polycystic ovary syndrome: a prospective randomized trial. GynecolEndocrinol. 2017 Nov 2;33(11):872- 76.

13. Kar S. Current evidence supporting "letrozole" for ovulation induction. J Hum Reprod Sci. 2013;6(2):93.

14. Seyedoshohadaei F. Comparison of the Effect of Clomiphene- EstradiolValerate vs Letrozole on Endometrial Thickness, Abortion and Pregnancy Rate in Infertile Women with Polycystic Ovarian Syndrome. J ClinDiagn Res 2016;10(8):QC10-3

15. Banerjee Ray P, Ray A, Chakraborti PS. Comparison of efficacy of letrozole and clomiphene citrate in ovulation induction in Indian women with polycystic ovarian syndrome. Arch Gynecol Obstet. 2012 Mar;285(3):873-77.

16. Franik S, Eltrop SM, Kremer JA, Kiesel L, Farquhar C. Aromatase inhibitors (letrozole) for subfertile women with polycystic ovary syndrome. Cochrane Database Syst Rev 2018,Issue 5.Art No.: CD010287. DOI:10.1002/14651858.pub3

17. Wang R, Li W, Bordewijk EM, Legro RS, Zhang H, Wu X, et al. First-line ovulation induction for polycystic ovary syndrome: an individual participant data meta-analysis. Hum Reprod Update. 2019 Nov $5 ; 25(6): 717-32$.

18. The Thessaloniki ESHRE/ASRM-Sponsored PCOS Consensus Workshop Group. Consensus on infertility treatment related to polycystic ovary syndrome. Hum Reprod. 2008;23:462-477.

19. Wang CF, Gemzell C. The use of human gonadotropins for the induction of ovulation in women with polycystic ovarian disease.FertilSteril. 1980;33:479-486.

20. Hajishafiha M, Dehghan M, Kiarang N, Sadegh-Asadi N, Shayegh SN, Ghasemi-Rad M. Combined letrozole and clomiphene versus letrozole and clomiphene alone in infertile patients with polycystic ovary syndrome. Drug Des DevelTher 2013;7:1427-31.

\section{Tables :}

Table -1

\section{Baseline characteristics of patients:}

\begin{tabular}{llll}
\hline characteristics & Letrozole + Placebo group $(\mathrm{n}=40)$ & Letrozole + CC group $(\mathrm{n}=40)$ & P-value \\
\hline${ }^{\mathrm{a}}$ Age & $28.07 \pm 3.22$ & $27.6 \pm 3.31$ & 0.5297 \\
${ }^{\mathrm{a}}$ Weight & $71.86 \pm 10.88$ & $71.33 \pm 10.53$ & 0.8286 \\
${ }^{\mathrm{a}}$ Height & $158.13 \pm 5.27$ & $156.3 \pm 4.74$ & 0.1131
\end{tabular}




\begin{tabular}{lllc}
\hline characteristics & Letrozole + Placebo group $(\mathrm{n}=40)$ & Letrozole + CC group $(\mathrm{n}=40)$ & P-value \\
\hline${ }^{\mathrm{a}}$ BMI & $28.66 \pm 3.52$ & $29.09 \pm 3.42$ & 0.5883 \\
${ }^{\mathrm{c}}$ secondary infertility & $12 / 40(30 \%)$ & $9 / 40(22.5 \%)$ & 0.612 \\
${ }^{\mathrm{c}}$ primary infertility & $28 / 40(70 \%)$ & $31 / 40(77.5 \%)$ & 0.612 \\
a Duration of infertility & $3.65 \pm 1.67$ & $4.12 \pm 1.6$ & 0.2111 \\
${ }^{\mathrm{b}}$ mFGS & $4(3-6)$ & $5(3-7)$ & 0.8571 \\
${ }^{\mathrm{a}}$ Day2 LH & $12.23 \pm 3.06$ & $11.45 \pm 2.73$ & 0.2413 \\
aDAY 2 FSH & $5.44 \pm 1.88$ & $5.41 \pm 2.31$ & 0.9504 \\
${ }^{\mathrm{a}}$ AMH & $5.79 \pm 2.16$ & $5.96 \pm 2.49$ & 0.7501 \\
d Phenotypes & & & \\
Phenotype A & $24(60 \%)$ & $27(67.5 \%)$ & 1.000 \\
Phenotype B & $8(20 \%)$ & $6(15 \%)$ & \\
Phenotype C & $5(12.5 \%)$ & $4(10 \%)$ & \\
Phenotype D & $3(7.5 \%)$ & $3(7.5 \%)$ & \\
\hline
\end{tabular}

Numerical data presented as mean \pm standard deviation; b. mFGS; modified Ferrimangallwey score presented as median (Interquartile range); c. Categorical data presented as n (\%); d. Phenotype $\mathrm{A}=\mathrm{AE}+\mathrm{OD}+\mathrm{PCOM}$, Phenotype $\mathrm{B}=\mathrm{AE}+\mathrm{OD}$, Phenotype $\mathrm{C}=\mathrm{AE}+\mathrm{PCOM}$, Phenotype $\mathrm{D}=\mathrm{OD}+\mathrm{PCOM}$ (AE- androgen excess, OD - ovarian dysfunction, PCOM - polycystic ovarian morphology).

Table 2

Intention to treat analysis (ITT):

Reproductive outcomes:

\begin{tabular}{llll}
\hline Outcome & Letrozole + Placebo group & Letrozole + CC group & Absolute d \\
\hline Intention-to-treat analysis/ primary outcome Ovulation & $15 / 40(37.5 \%)$ & $29 / 40(72.5 \%)$ & $35(14.59$ tc \\
Secondary outcomes & & & \\
Conception & $3 / 40(7.5 \%)$ & $4 / 40(10 \%)$ & $2.5(-9.87 \%$ \\
Clinical pregnancy & $2 / 40(5 \%)$ & $3 / 40(7.5 \%)$ & $2.5(-8.09 \%$ \\
Multiple Pregnancy & 0 & 0 & - \\
Pregnancy loss among those who conceived & $1 / 40(2.5 \%)$ & $1 / 40(2.5 \%)$ & 0 \\
\hline
\end{tabular}

Table 3

Per protocol analysis (PP):

Reproductive outcomes:

\begin{tabular}{llll}
\hline Outcome & Letrozole + Placebo group & Letrozole + CC group & Absolute differe \\
\hline Per-protocol analysis/ primary outcome Ovulation & $15 / 38(39.4 \%)$ & $29 / 39(74.35 \%)$ & $34.95(14.17 \%$ to \\
Secondary outcomes & & & \\
Conception & $3 / 38(7.89 \%)$ & $4 / 39(10.25 \%)$ & $2.36(-10.45 \%$ to \\
Clinical pregnancy & $2 / 38(5.26 \%)$ & $3 / 39(7.69 \%)$ & $2.43(-8.54 \%$ to \\
Multiple Pregnancy & 0 & 0 & 0 \\
Pregnancy loss among those who conceived & $1 / 38(2.63 \%)$ & $1 / 39(2.56 \%)$ & $0.07(-7.04 \%$ to \\
\hline
\end{tabular}

Table-4 
Cycle characteristics

\begin{tabular}{lll}
\hline & Letrozole + Placebo group & Letrozo \\
\hline No. of follicles $>15 \mathrm{~mm} /$ cycle & $0.94 \pm 0.99$ & $1.56 \pm 1$. \\
No. of women with follicles $>15 \mathrm{~mm}$ & $21 / 38$ & $33 / 39$ \\
Endometrial lining thickness, mm & $8.12 \pm 1.92$ & $8.18 \pm 1$. \\
Cycle characteristics among those who ovulated & Cycle characteristics among those who ovulated & Cycle \\
No. of follicles $>15 \mathrm{~mm} /$ Cycle & $1.46 \pm 0.61$ & $1.9 \pm 0.8$ \\
Endometrial lining thickness, mm & $8.57 \pm 1.54$ & $8.67 \pm 1$. \\
\hline
\end{tabular}

Table 5

Adverse events

Letrozole + Placebo group $(\mathrm{n}=39) \quad$ Letrozole $+\mathrm{C}$

\begin{tabular}{lll}
\hline Side-effects acceptability & & \\
Acceptable & $15 / 39(38.4 \%)$ & $18 / 38(47.3 \%)$ \\
Not acceptable & $0 / 39(0)$ & $0 / 38(0)$ \\
No side effects & $24 / 39(61.5 \%)$ & $20 / 38(52.7 \%)$ \\
Hospitalised or seek any additional care during the treatment month. & $0 / 39$ & $0 / 38$ \\
Side-effects during treatment month (before conception) & & $0 / 40$ \\
Serious adverse events & $0 / 40$ & $18 / 38(47.3 \%)$ \\
Reported side-effects & $15 / 39(38.4 \%)$ & $11 / 38(28.9 \%)$ \\
Headache & $10 / 39(25.6 \%)$ & $6 / 38(15.6 \%)$ \\
Hot flush & $2 / 39(5.1 \%)$ & $8 / 38(21 \%)$ \\
Abdominal bloating & $7 / 39(17.9 \%)$ & $4 / 38(10.4 \%)$ \\
Abdominal cramp & $4 / 39(10.2 \%)$ & $6 / 38(15.6 \%)$ \\
Nausea & $5 / 39(12.8 \%)$ & $4 / 38(10.4 \%)$ \\
Mood changes & $5 / 39(12.8 \%)$ & $5 / 38(13.1 \%)$ \\
Fatigue & $6 / 39(15.3 \%)$ & $4 / 38(10.4 \%)$ \\
Back pain & $5 / 39(12.8 \%)$ & $1 / 38(2.6 \%)$ \\
Dizziness & $2 / 39(5.1 \%)$ & $5 / 38(13.1 \%)$ \\
Breast discomfort & $3 / 39(7.6 \%)$ & $2 / 38(5.2 \%)$ \\
Diarrhoea & $1 / 39(2.5 \%)$ & \\
\hline
\end{tabular}

Figure 1: Participant flow diagram according to Consolidated Standards of Reporting Trials (CONSORT). 
Participant Flow Diagram

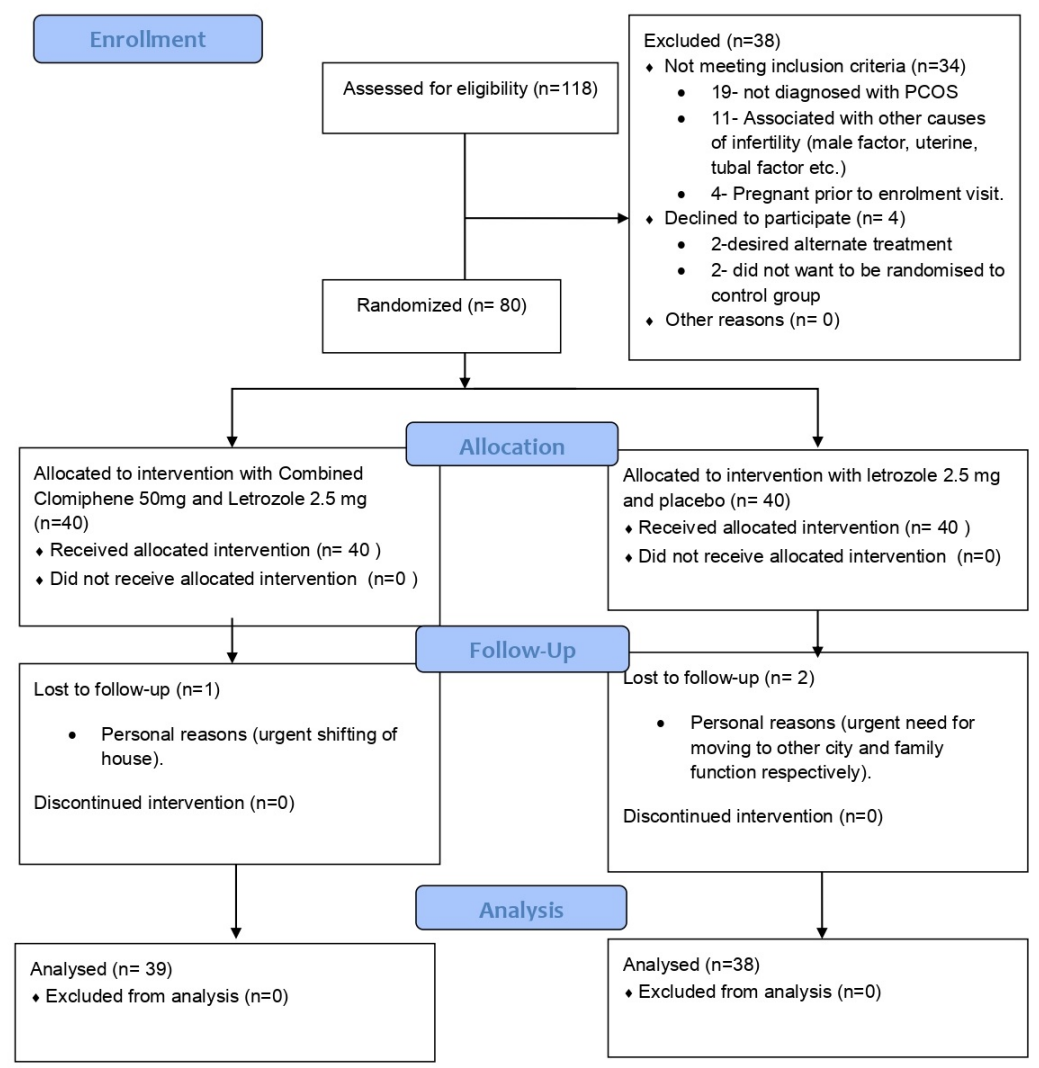

\section{Hosted file}

fgure.docx available at https://authorea.com/users/421961/articles/527787-a-single-centredouble-arm-triple-blind-randomised-controlled-trial-comparing-the-efficacy-of-combinedletrozole-and-clomiphene-versus-the-only-letrozole-as-a-method-of-ovulation-inductionin-women-with-polycystic-ovarian-syndrome 